\title{
Model Order Reduction for Elastic Multibody Systems with Fast Rotating Flexible Bodies
}

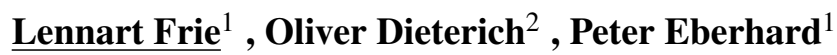 \\ ${ }^{1}$ Institute of Engineering and Computational \\ Mechanics, University of Stuttgart \\ Pfaffenwaldring 9, 70569 Stuttgart, Germany \\ lennart.frie@itm.uni-stuttgart.de \\ peter.eberhard@itm.uni-stuttgart.de \\ ${ }^{2}$ Airbus Helicopters Germany $\mathrm{GmbH}$ \\ Dynamics and Vibrations \\ Industriestr. 4, 86607 Donauwörth, Germany \\ oliver.dieterich@airbus.com
}

\begin{abstract}
The dynamical behavior of Elastic Multibody Systems (EMBS) is often analyzed using virtual prototypes described by high-dimensional systems of differential equations. Model Order Reduction (MOR) is a key step to permit efficient system evaluations by approximating the full system with a reduced order surrogate model. It is one challenge in MOR of EMBS, to describe the dynamics induced through the coupling of bodies in the reduced system. In this contribution, a workflow for the reduction of EMBS with fast rotating bodies is presented. The rotation causes a change of dynamical behavior due to inertia forces and, therefore, cannot be neglected. In the scope of this work a linear description of rotating bodies with constant angular velocity is given. Different projection-based MOR techniques are compared and applied to an industrial model of a helicopter with rotating rotor. For this purpose, a short introduction on modeling of EMBS and MOR is given. Substructured reduction is then contrasted to the reduction of the coupled system for modal reduction techniques, moment matching based on Krylov subspaces, and Proper Orthogonal Decomposition. The approximation errors of the reduced systems are compared in frequency domain. It is shown that rotation-dependent terms are essential to describe the dynamic behavior of the system correctly. Reduced models with low approximation errors and large speed-up are obtained with substructured Proper Orthogonal Decomposition and outperform the standard techniques modal truncation and Craig-Bampton reduction.
\end{abstract}

Keywords: Elastic Multibody System, Rotating Body, Model Order Reduction, Rotor Dynamics.

\section{INTRODUCTION}

Elastic Multibody Systems (EMBS) are often simulated to study the dynamical behavior of complex mechanical systems. High complexity of the underlying models and increasing demands on details make the use of Model Order Reduction (MOR) inevitable. MOR aims at generating reduced models that allow numerically efficient system evaluations with a small approximation error in the mathematically defined space of interest. Classical reduction approaches in the field of EMBS, e.g., the Craig-Bampton-Method (CBM) [1], reduce the different bodies separately with modal MOR techniques and then build the reduced system by assembling the reduced bodies. However, these standard methods can not always account correctly for the interaction between the different bodies. Nevertheless, modal approaches are still state of the art. Input-output based MOR techniques, instead, are often better suited for coupled systems [2]. This contribution extends existing MOR methods for usage for EMBS with fast rotating bodies where additional, rotationdependent terms have to be added to the equations of motion. Furthermore, the applicability to an industrial use case, here a helicopter model with non-rotating flexible airframe and rotating main rotor, is demonstrated and the reduced order systems are compared. The novelty of the contribution is the application of existing MOR methods to EMBS with fast rotating bodies in a workflow that is applicable to large scale systems. 


\section{THEORETICAL BACKGROUND}

\subsection{Elastic Multibody Systems with Rotating Bodies}

In computational mechanics, EMBS are often described with the floating frame of reference approach, see e.g. [3]. The idea is to separate the motion of the system into large nonlinear described rigid body motion and small linear described elastic deformations with respect to a moving reference frame that is rigidly attached to the body. The equations of motion of an EMBS can then be written as

$$
\underbrace{\left[\begin{array}{cc}
\boldsymbol{M}_{\mathrm{r}} & \boldsymbol{M}_{\mathrm{re}} \\
\boldsymbol{M}_{\mathrm{er}} & \boldsymbol{M}_{\mathrm{e}}
\end{array}\right]}_{=: \boldsymbol{M}}\left[\begin{array}{c}
\ddot{\boldsymbol{q}}_{\mathrm{r}} \\
\ddot{\boldsymbol{q}}_{\mathrm{e}}
\end{array}\right]=\boldsymbol{h},
$$

where the mass matrix $\boldsymbol{M}$ is separated into a submatrix $\boldsymbol{M}_{\mathrm{r}}$ which describes the rigid body dynamics, a submatrix $\boldsymbol{M}_{\mathrm{e}}$ which is a block-diagonal matrix containing all element mass matrices of the elastic bodies, and the coupling matrices $\boldsymbol{M}_{\mathrm{re}}$ and $\boldsymbol{M}_{\mathrm{er}}$ with $\boldsymbol{M}_{\mathrm{re}}=\boldsymbol{M}_{\mathrm{er}}^{\mathrm{T}}$. The vectors $\boldsymbol{q}_{\mathrm{r}}$ and $\boldsymbol{q}_{\mathrm{e}}$ are the rigid body coordinates and the elastic coordinates, respectively. In this work only rotational rigid body motion is considered. The force vector $\boldsymbol{h}$ summarizes Coriolis, constraint, centrifugal, inner and external forces.

A tool often used to describe small elastic deformations of a single body $k$ of the system is the linear Finite Element Method (FEM). The equations of motion of the free body $k$ then read

$$
\boldsymbol{M}_{\mathrm{e}}^{\mathrm{k}} \ddot{\boldsymbol{q}}_{\mathrm{e}}^{\mathrm{k}}+\boldsymbol{K}_{\mathrm{e}}^{\mathrm{k}} \boldsymbol{q}_{\mathrm{e}}^{\mathrm{k}}=\boldsymbol{f}^{\mathrm{k}}
$$

with external forces $\boldsymbol{f}^{\mathrm{k}}$ and the linear material stiffness matrix $\boldsymbol{K}_{\mathrm{e}}^{\mathrm{k}}$ that describes the linear part of inner forces $\boldsymbol{k}_{\mathrm{e}}^{\mathrm{k}}$ of the body resulting from virtual work of inner forces

$$
\boldsymbol{k}_{\mathrm{e}}^{\mathrm{k}} \boldsymbol{\delta} \boldsymbol{q}_{\mathrm{e}}^{\mathrm{k}}=\int_{V_{0}^{\mathrm{k}}} \boldsymbol{\delta} \dot{\boldsymbol{\varepsilon}}^{\mathrm{k}^{\mathrm{T}}} \boldsymbol{\sigma}^{\mathrm{k}} \mathrm{dV} .
$$

The distortion velocities $\dot{\boldsymbol{\varepsilon}}^{\mathrm{k}}$ and the stress $\boldsymbol{\sigma}^{\mathrm{k}}$ in Equation (3) can be expressed with the distortion matrices $\boldsymbol{B}_{\mathrm{L}}^{\mathrm{k}}, \boldsymbol{B}_{\mathrm{N}}^{\mathrm{k}}$ and material matrix $\boldsymbol{H}^{\mathrm{k}}$ by

$$
\boldsymbol{\delta} \dot{\boldsymbol{\varepsilon}}^{\mathrm{k}}=\left(\boldsymbol{B}_{\mathrm{L}}^{\mathrm{k}}+\boldsymbol{B}_{\mathrm{N}}^{\mathrm{k}}\right) \boldsymbol{\delta} \dot{\boldsymbol{q}}^{\mathrm{k}}, \quad \boldsymbol{\sigma}^{\mathrm{k}}=\boldsymbol{\sigma}_{0}^{\mathrm{k}}+\boldsymbol{H}^{\mathrm{k}}\left(\boldsymbol{B}_{\mathrm{L}}^{\mathrm{k}}+\frac{1}{2} \boldsymbol{B}_{\mathrm{N}}^{\mathrm{k}}\right),
$$

see e.g. [4]. Here, $\boldsymbol{\sigma}_{0}^{\mathrm{k}}$ are pre-stresses in the body and subscripts ' $L$ ' and ' $N$ ' denote linear and nonlinear parts of $\boldsymbol{B}^{\mathrm{k}}$, respectively. For a stress-free reference configuration, $\boldsymbol{\sigma}_{0}^{\mathrm{k}}=\mathbf{0}$, the inner forces of the body $k$ can be written as

$$
\boldsymbol{k}_{\mathrm{e}}^{\mathrm{k}}=\underbrace{\int_{V_{0}^{\mathrm{k}}} \boldsymbol{B}_{\mathrm{L}}^{\mathrm{k}^{\mathrm{T}}} \boldsymbol{H}^{\mathrm{k}} \boldsymbol{B}_{\mathrm{L}}^{\mathrm{k}} \mathrm{dV}}_{=\boldsymbol{K}_{\mathrm{e}}^{\mathrm{k}} \boldsymbol{q}_{\mathrm{e}}^{\mathrm{k}}}+\underbrace{\int_{V_{0}^{\mathrm{k}}} \boldsymbol{B}_{\mathrm{N}}^{\mathrm{k}^{\mathrm{T}}} \boldsymbol{H}^{\mathrm{k}} \boldsymbol{B}_{\mathrm{L}}^{\mathrm{k}} \mathrm{dV}}_{\text {III }}+\underbrace{\frac{1}{2} \int_{V_{0}^{\mathrm{k}}}\left(\boldsymbol{B}_{\mathrm{L}}^{\mathrm{k}}+\boldsymbol{B}_{\mathrm{N}}^{\mathrm{k}}\right)^{\mathrm{T}} \boldsymbol{H}^{\mathrm{k}} \boldsymbol{B}_{\mathrm{N}}^{\mathrm{k}} \mathrm{dV}}_{\text {IIII }} .
$$

With the assumption that $\boldsymbol{q}_{\mathrm{e}}, \dot{\boldsymbol{q}}_{\mathrm{e}}$ and $\ddot{\boldsymbol{q}}_{\mathrm{e}}$ are small, the nonlinear parts (II) and III in Equation (5) are neglected in linear FEM and only the first integral is kept in Equation (2).

However, the situation for fast rotating bodies is more complicated and usual linear FEM is not sufficient. If the body $k$ underlies fast rotations, it is loaded with large inertia forces

$$
\boldsymbol{h}_{\omega_{\mathrm{e}}}^{\mathrm{k}}=-\underbrace{2 \int_{V_{0}^{\mathrm{k}}} \boldsymbol{N}^{\mathrm{k}^{\mathrm{T}}} \tilde{\boldsymbol{\omega}}^{\mathrm{k}} \boldsymbol{N}^{\mathrm{k}} \dot{\boldsymbol{q}}_{\mathrm{e}}^{\mathrm{k}} \mathrm{dm}}_{=: \boldsymbol{G}_{\mathrm{e}}^{\mathrm{k}} \dot{\boldsymbol{q}}_{\mathrm{e}}^{\mathrm{k}}}-\underbrace{\int_{V_{0}^{\mathrm{k}}} \boldsymbol{N}^{\mathrm{k}^{\mathrm{T}}} \tilde{\boldsymbol{\omega}}^{\mathrm{k}} \tilde{\boldsymbol{\omega}}^{\mathrm{k}}\left(\boldsymbol{R}+\boldsymbol{N}^{\mathrm{k}} \boldsymbol{q}_{\mathrm{e}}^{\mathrm{k}}\right) \mathrm{dm}}_{=: \boldsymbol{K}_{\mathrm{s}}^{\mathrm{k}} \boldsymbol{q}_{\mathrm{e}}^{\mathrm{k}}}
$$


which add to $f^{\mathrm{k}}$ on the right side of Equation (2). Here, $\boldsymbol{N}^{\mathrm{k}}$ are the finite element ansatz functions, $\boldsymbol{R}$ is the position of a point of the undeformed body described in the reference frame, $\tilde{\boldsymbol{\omega}}^{\mathrm{k}}$ is a skew-symmetric matrix with

$$
\tilde{\boldsymbol{\omega}}^{\mathrm{k}}=\left[\begin{array}{ccc}
0 & -\omega_{3}^{\mathrm{k}} & \omega_{2}^{\mathrm{k}} \\
\omega_{3}^{\mathrm{k}} & 0 & -\omega_{1}^{\mathrm{k}} \\
-\omega_{2}^{\mathrm{k}} & \omega_{1}^{\mathrm{k}} & 0
\end{array}\right]
$$

and $\boldsymbol{\omega}^{\mathrm{k}}=\left[\omega_{1}^{\mathrm{k}}, \omega_{2}^{\mathrm{k}}, \omega_{3}^{\mathrm{k}}\right]^{\mathrm{T}}$ are the angular velocities of the body $k$. The matrices $\boldsymbol{G}_{\mathrm{e}}^{\mathrm{k}}$ and $\boldsymbol{K}_{\mathrm{s}}^{\mathrm{k}}$, defined in Equation (6), both depend on $\boldsymbol{\omega}^{\mathrm{k}}$ and are called gyroscopic matrix and spin softening matrix of the body $k$, see e.g. [5].

If the assumption of small deformations should be still justified, the large inertia forces must not result in large deformations, but only in large stress in the body. The stress is in linear approximation described by $\boldsymbol{\sigma}^{\mathrm{k}}=\boldsymbol{H}^{\mathrm{k}} \boldsymbol{B}_{\mathrm{L}}^{\mathrm{k}}$ and, thus, the integral (II) in Equation (5) is not small anymore. For this reason, an additional stiffness has to be considered in directions of large inertia forces. This so-called geometric stiffness depends on the stress in the body and, therefore, on the rotational velocity of the body. It can be added to the linear equation of motion of the body by expanding the integral (II) to a Taylor series at $\boldsymbol{q}_{\mathrm{e}}^{\mathrm{k}}=\mathbf{0}$ and just considering the terms linear in $\boldsymbol{q}_{\mathrm{e}}^{\mathrm{k}}$. This leads to the approximation

$$
\boldsymbol{K}_{\mathrm{geo}}^{\mathrm{k}} \boldsymbol{q}_{\mathrm{e}}^{\mathrm{k}} \approx \int_{V_{0}^{\mathrm{k}}} \boldsymbol{B}_{\mathrm{N}}^{\mathrm{k}^{\mathrm{T}}} \boldsymbol{H}^{\mathrm{k}} \boldsymbol{B}_{\mathrm{L}}^{\mathrm{k}} \mathrm{dV},
$$

where the matrix $\boldsymbol{K}_{\text {geo }}^{\mathrm{k}}$ is called geometric stiffness matrix. The linear approximation is only valid for a given nominal stress in the body and, thus, for a given nominal angular velocity. For this specific velocity, the updated equations of motion of a rotating body then read

$$
\boldsymbol{M}_{\mathrm{e}}^{\mathrm{k}} \ddot{\boldsymbol{q}}_{\mathrm{e}}^{\mathrm{k}}+\boldsymbol{G}_{\mathrm{e}}^{\mathrm{k}} \dot{\boldsymbol{q}}_{\mathrm{e}}^{\mathrm{k}}+\underbrace{\left(\boldsymbol{K}_{\mathrm{e}}^{\mathrm{k}}+\boldsymbol{K}_{\mathrm{geo}}^{\mathrm{k}}+\boldsymbol{K}_{\mathrm{s}}^{\mathrm{k}}\right)}_{=: \hat{\boldsymbol{K}}_{\mathrm{e}}^{\mathrm{k}}} \boldsymbol{q}_{\mathrm{e}}^{\mathrm{k}}=\boldsymbol{f}_{\mathrm{e}}^{\mathrm{k}}
$$

with $\boldsymbol{G}_{\mathrm{e}}^{\mathrm{k}}$ and $\boldsymbol{K}_{\mathrm{s}}^{\mathrm{k}}$ from Equation (6), $\boldsymbol{K}_{\mathrm{geo}}^{\mathrm{k}}$ from Equation (8), and the updated stiffness matrix $\hat{\boldsymbol{K}}_{\mathrm{e}}^{\mathrm{k}}$. For a detailed explanation see [4].

With the description of the separate bodies the equations of motion of the coupled system can now be set up. A suitable description of a coupled EMBS is the formulation in minimal coordinates $\boldsymbol{q} \in \mathbb{R}^{N}$ with

$$
\left[\begin{array}{l}
\boldsymbol{q}_{\mathrm{r}} \\
\boldsymbol{q}_{\mathrm{e}}
\end{array}\right]=\boldsymbol{J} \boldsymbol{q}
$$

where $\boldsymbol{J}$ is the global Jacobian matrix of the system, see e.g. [6]. The coupled EMBS is then described by

$$
\boldsymbol{J}^{\mathrm{T}} \boldsymbol{M} \boldsymbol{J} \ddot{\boldsymbol{q}}+\boldsymbol{J}^{\mathrm{T}}\left[\begin{array}{cc}
\mathbf{0} & \mathbf{0} \\
\mathbf{0} & \boldsymbol{G}_{\mathrm{e}}
\end{array}\right] \boldsymbol{J} \dot{\boldsymbol{q}}+\boldsymbol{J}^{\mathrm{T}}\left[\begin{array}{cc}
\mathbf{0} & \mathbf{0} \\
\mathbf{0} & \hat{\boldsymbol{K}}_{\mathrm{e}}
\end{array}\right] \boldsymbol{J} \boldsymbol{q}=\boldsymbol{J}^{\mathrm{T}}\left[\begin{array}{c}
\boldsymbol{h}_{\mathrm{r}} \\
\boldsymbol{f}_{\mathrm{e}}
\end{array}\right]
$$

where $\boldsymbol{M}_{\mathrm{e}}$ in $\boldsymbol{M}, \boldsymbol{G}_{\mathrm{e}}$ and $\hat{\boldsymbol{K}}_{\mathrm{e}}$ are block-diagonal matrices containing the corresponding matrices of the different bodies, $\boldsymbol{h}_{\mathrm{r}}$ are the rigid body forces, and $\boldsymbol{f}_{\mathrm{e}}$ are the external forces applied to the nodes of the elastic bodies.

The matrix $\boldsymbol{J}$ depends on the orientation of the reference systems of the bodies and, therefore, Equation (11) is nonlinear. For small rotations around a reference orientation, it can be linearized with small-angle approximation and neglect of terms nonlinear in $\boldsymbol{q}$. The linearized equation of motion of the system then reads

$$
\boldsymbol{M}_{\mathrm{L}} \ddot{\boldsymbol{q}}+\boldsymbol{G}_{\mathrm{L}} \dot{\boldsymbol{q}}+\boldsymbol{K}_{\mathrm{L}} \boldsymbol{q}=\boldsymbol{f}_{\mathrm{L}}
$$


but includes certain terms due to fast rotations. The index ' $\mathrm{L}$ ' is omitted in the following but we always refer to the linearized system from here on. It can be interpreted as second order system

$$
\begin{aligned}
M \ddot{q}+G \dot{q}+K \boldsymbol{q} & =B \boldsymbol{B}, \\
y & =C \boldsymbol{q}
\end{aligned}
$$

with system matrices $\boldsymbol{M}, \boldsymbol{G}, \boldsymbol{K} \in \mathbb{R}^{N \times N}, \boldsymbol{B} \in \mathbb{R}^{N \times b}, \boldsymbol{C} \in \mathbb{R}^{c \times N}$ inputs $\boldsymbol{u} \in \mathbb{R}^{b}$ and outputs $\boldsymbol{y} \in \mathbb{R}^{c}$.

\subsection{Projection-Based Model Order Reduction}

The dimension $N$ of the system in Equation (13) is usually high and system evaluations are numerically expensive. It is the goal of MOR, to approximate the full order system with a low order system while simultaneously ensuring a low approximation error. In projection-based MOR this is done by the projection of the state vector

$$
\boldsymbol{q} \approx \boldsymbol{V} \tilde{\boldsymbol{q}}, \quad \operatorname{dim}(\boldsymbol{q})=N \gg \operatorname{dim}(\tilde{\boldsymbol{q}})=n
$$

into a low dimensional subspace $\mathscr{V}=\operatorname{span}\{\boldsymbol{V}\}$ with the projection matrix $\boldsymbol{V} \in \mathbb{R}^{N \times n}$. Plugging this into Equation (13) and left-multiplying with $\boldsymbol{V}^{\mathrm{T}}$ yields the reduced order model

$$
\begin{aligned}
\underbrace{\boldsymbol{V}^{\mathrm{T}} \boldsymbol{M} \boldsymbol{V}}_{=: \tilde{\boldsymbol{M}}} \ddot{\tilde{\boldsymbol{q}}}+\underbrace{\boldsymbol{V}^{\mathrm{T}} \boldsymbol{G} \boldsymbol{V}}_{=: \tilde{\boldsymbol{G}}} \dot{\tilde{\boldsymbol{q}}}+\underbrace{\boldsymbol{V}^{\mathrm{T}} \boldsymbol{K} \boldsymbol{V}}_{=: \tilde{\boldsymbol{K}}} \tilde{\boldsymbol{q}}=\underbrace{\boldsymbol{V}^{\mathrm{T}} \boldsymbol{B}}_{=: \tilde{\boldsymbol{B}}} \boldsymbol{u}, \\
\tilde{\boldsymbol{y}}=\underbrace{\boldsymbol{C} \boldsymbol{\boldsymbol { q }}}_{=: \tilde{\boldsymbol{C}}} .
\end{aligned}
$$

The choice of $\boldsymbol{V}$ is the key challenge here, since there are two contrary requirements, i.e. that the reduced system dimension shall be small, $n \ll N$, and the approximation error is requested to be low. Classical modal approaches propose finding $\boldsymbol{V}$ by taking selected dominant eigenmodes $\boldsymbol{V}=\left[\boldsymbol{\phi}_{1}, \ldots, \boldsymbol{\phi}_{n}\right]$ of the system as its columns.

A second approach is to approximate the input-output behavior of the system. Therefore, in Krylov reduction the transfer function $\boldsymbol{H}(s)$ of Equation (13) is written as a power series where $s$ is the Laplace variable. Now, the terms of the power series of the reduced system, that are also called moments, are matched up to a defined order $J_{l}$ around shifts in frequency domain $s=\sigma_{l}$ with those of the full system. The moments are not calculated explicitly, but are implicitly matched by the use of Krylov subspaces. A numerically stable algorithm to produce such subspaces is the second order Arnoldi (SOAR) algorithm, explained in [7] and [8]. Choosing the projection matrix for $J_{l}=1$ with

$$
\operatorname{span}(\boldsymbol{V})=\operatorname{span}\left[\left(\sigma_{1}^{2} \boldsymbol{M}+\sigma_{1} \boldsymbol{G}+\boldsymbol{K}\right)^{-1} \boldsymbol{B}, \quad \ldots, \quad\left(\sigma_{v}^{2} \boldsymbol{M}+\sigma_{v} \boldsymbol{G}+\boldsymbol{K}\right)^{-1} \boldsymbol{B}\right]
$$

for $l=1, \ldots, v$ ensures

$$
\boldsymbol{H}\left(\sigma_{l}\right)=\tilde{\boldsymbol{H}}\left(\sigma_{l}\right)
$$

and if $\boldsymbol{C}=\boldsymbol{B}^{\mathrm{T}}$,

$$
\frac{\partial \boldsymbol{H}\left(\sigma_{l}\right)}{\partial s}=\frac{\partial \tilde{\boldsymbol{H}}\left(\sigma_{l}\right)}{\partial s},
$$

as [9] shows. In this basic form, the order of the reduced model is always a multiple of the number of inputs $b=\operatorname{dim}(\boldsymbol{u})$.

A third approach on generating $\boldsymbol{V}$ is based on balanced truncation, see e.g. [10]. It aims at retaining just those states in the reduced system that are easy to reach and easy to observe, i.e., those that require little energy to be reached and yield large observation signals when excited. The computation of the Gramian matrices that are required to detect those states is numerically 
expensive and, therefore, not practicable for large-scale systems. However, the Gramian matrix of controllability

$$
\boldsymbol{P}=\frac{1}{\pi} \int_{s_{\min }}^{s_{\max }} \hat{\boldsymbol{F}}(s) \hat{\boldsymbol{F}}(s)^{\mathrm{T}} \mathrm{ds}
$$

with

$$
\hat{\boldsymbol{F}}(s):=[\operatorname{Re}(\boldsymbol{F}(s)), \operatorname{Im}(\boldsymbol{F}(s))] \in \mathbb{R}^{\mathrm{N} \times 2 \mathrm{~b}}, \quad \boldsymbol{F}(s)=\left(s^{2} \boldsymbol{M}+s \boldsymbol{G}+\boldsymbol{K}\right)^{-1} \boldsymbol{B} \in \mathbb{C}^{\mathrm{N} \times \mathrm{b}}
$$

can be approximated with Proper Orthogonal Decomposition (POD). Therefore, $v$ different snapshots $\hat{\boldsymbol{F}}\left(\sigma_{l}\right)$ are computed at frequencies $s=\sigma_{l}$ for $l=1, \ldots, v$ and these snapshots are combined to

$$
\boldsymbol{Z}=\left[\hat{\boldsymbol{F}}\left(\sigma_{1}\right), \hat{\boldsymbol{F}}\left(\sigma_{2}\right), \ldots, \hat{\boldsymbol{F}}\left(\sigma_{v}\right)\right] \in \mathbb{R}^{N \times 2 v b} .
$$

The projection matrix is then build with the first $n$ eigenvectors $v_{\mathrm{i}} \in \mathbb{R}^{\mathrm{N}}, i=1,2, \ldots, n$ from the eigenvalue problem

$$
\frac{1}{2 v b} Z Z^{\mathrm{T}} \boldsymbol{v}_{\mathrm{i}}=\lambda_{\mathrm{i}} \boldsymbol{v}_{\mathrm{i}}
$$

and reads $\boldsymbol{V}=\left[\boldsymbol{v}_{1}, \boldsymbol{v}_{2}, \ldots, \boldsymbol{v}_{n}\right]$. For further details on Gramian matrices and POD, see [10] and [11].

\section{REDUCTION OF ELASTIC MULTIBODY SYSTEMS WITH ROTATING BODIES}

A workflow for the reduction of EMBS is outlined in Figure 1. We start with discretized flexible bodies $k=1, \ldots, \kappa$ as used in FEM in the upper box. For the sake of simplicity, just two bodies are illustrated here, but the procedure is also applicable for an EMBS with $\kappa>2$ bodies. The equations of motion of the free bodies are formulated with linear FEM. The marked points are the coupling points that have to be defined.

If a body $k$ underlies fast rotations, additional terms in the linear equation of motion have to be considered. Therefore, the inertia forces are computed for a given constant rotational velocity vector $\boldsymbol{\omega}_{\mathrm{c}}^{\mathrm{k}}$. In this work the multibody simulation tool Neweul-M ${ }^{2}$ [12] is used for the computation of the inertia forces. The assumption of a constant rotational velocity is valid for many use cases and necessary in order to obtain a linear description that represents the nonlinear rotation-dependent effects so that this linear description can be used for linear MOR. With the constant inertia forces, also the gyroscopic matrix $\boldsymbol{G}_{\mathrm{e}}^{\mathrm{k}}$ and the spin softening matrix $\boldsymbol{K}_{\mathrm{s}}^{\mathrm{k}}$ are obtained.

With the constant inertia forces $\boldsymbol{h}_{\omega_{\mathrm{e}}}^{\mathrm{k}}\left(\boldsymbol{\omega}_{\mathrm{c}}^{\mathrm{k}}\right)$, the geometric stiffness matrix $\boldsymbol{K}_{\mathrm{geo}}^{\mathrm{k}}$ can be computed iteratively from the equilibrium $\boldsymbol{h}_{\omega_{\mathrm{e}}}^{\mathrm{k}}\left(\boldsymbol{\omega}_{\mathrm{c}}^{\mathrm{k}}\right)=\boldsymbol{k}_{\mathrm{e}}^{\mathrm{k}}$ with a nonlinear static finite-element solver, here MSC Nastran [13]. If the EMBS contains different rotating bodies, this procedure is carried out for each of them and Equation (9) is obtained for each body. Up to here all elastic bodies are treated separately.

The reduced model of the EMBS can now be calculated with two different strategies introduced in [14]. One, illustrated on the left side of Figure 1, is to first couple the system and thereafter reduce the coupled system with one of the methods proposed in Subsection 2.2. This workflow is in the following referred to as block structure preserving reduction (BSPR). However, another procedure that is often beneficial for the reduction of multibody systems is to divide the system into substructures, e.g. the individual bodies of a system with $\kappa$ bodies, first and then build reduced bases separately for these bodies. The reduced bodies are afterwards coupled to obtain the reduced coupled system. This approach is in the following called separate bases reduction (SBR) and visualized with the right path in Figure 1. The reduced bodies are graphically indicated by sparser grids. Reduced separate substructure models have various advantages. They are often easier to validate [15] and provide the possibility of independent component optimization. In addition, substructured models are useful in modern product development where different departments work on different components [14] because substructures can be changed without having to reduce the 


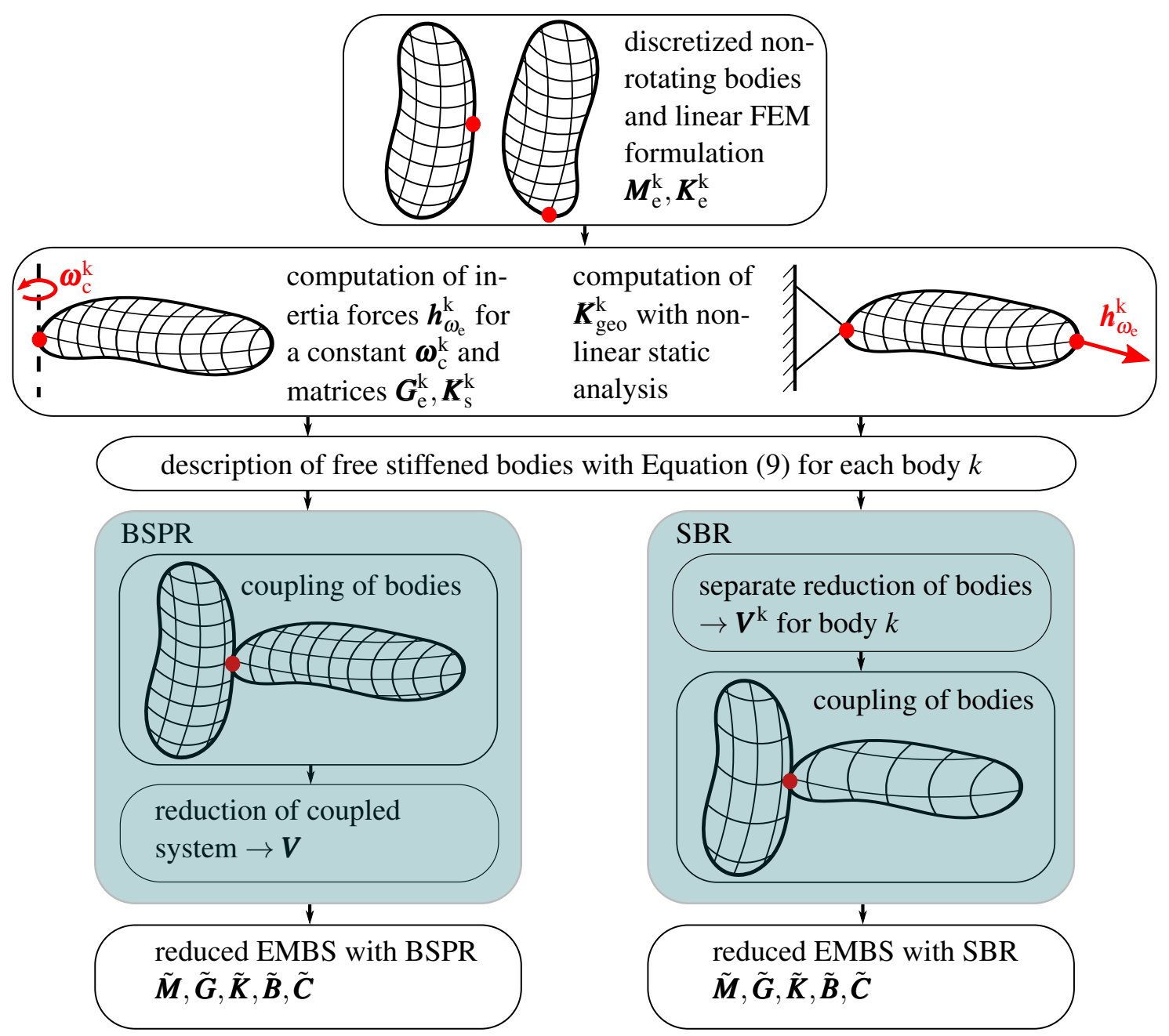

Figure 1: Workflow for the reduction of EMBS with rotating bodies

whole system again. One of the most frequently used SBR methods is the CBM introduced in [1]. The CBM is an extension of simple modal truncation and combines eigenmodes of the body with static constraint modes that define displacements due to unit forces at boundary nodes where the bodies are connected.

\section{NUMERICAL EXAMPLE}

The workflow presented in Section 3 is now applied to an industrial helicopter model as shown in Figure 2. The model of the airframe and the model of the rotor result from discretization for FEM and have 281992 and 3726 degrees of freedom (dof), respectively. A typical problem here is the approximation of the airframe with a low-rank model which can account for interactions with a coupled rotor. This is needed, e.g., for multi-domain simulations with aerodynamic rotor simulation tools. A basic modal truncation of the airframe model without considering the rotor leads to a reduced model that neglects important modes, e.g., shaft-bending modes. To counteract this, it is the state of the art approach to attach a surrogate mass that replaces the rotor inertia as explained in [16]. The loaded model is then reduced with modal truncation and the added surrogate mass is removed later by the use of negative boundary conditions in the coupling process. It is a problem with this procedure to find an adequately set up surrogate mass to approximate the inertia of a rotating rotor.

During operation, the rotor rotates with a constant velocity. This allows the application of the 


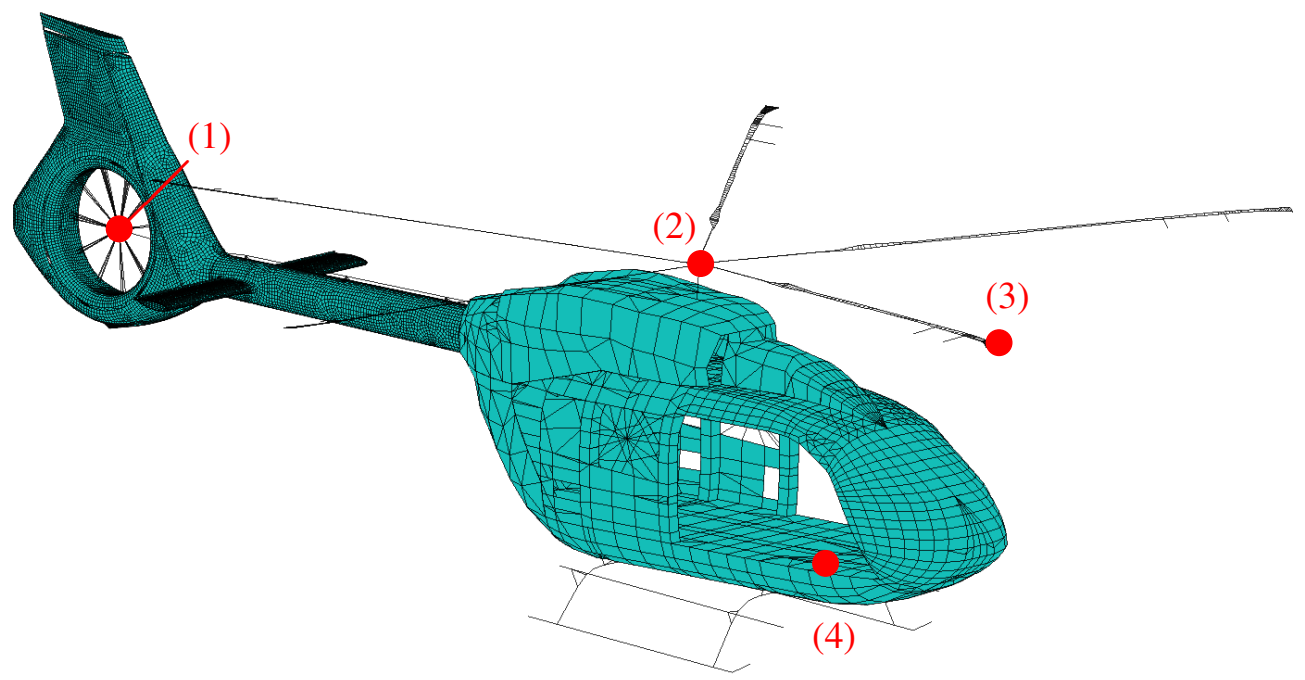

Figure 2: Model of a helicopter airframe with coupled rotor and marked inputs and outputs (model provided by Airbus Helicopters)

procedure described in Section 3 to obtain an appropriate linear model. The difference between the rotor modeled with linear FEM alone and with the additional rotation-dependent terms introduced in Subsection 2.1 is shown in Figure 3.

The Campbell Diagram in Figure 3a illustrates the change of blade eigenfrequencies over increasing angular velocities. Here, the first 9 eigenfrequencies are shown over the angular velocity subjected to the nominal operational velocity. The linear equation of motion of a rotating body is only valid for a constant rotational velocity. Thus, the Campbell Diagram results from computation of the rotation dependent terms at 50 linearly spaced frequencies in the interval $[0,140] \% \cdot f_{\text {op }}$ with nominal operation frequency $f_{\text {op }}$. Some eigenfrequencies are strongly shifted, e.g., the first eigenfrequency from $1.14 \mathrm{~Hz}$ in the non-rotating case to $6.69 \mathrm{~Hz}$ for rotation with $f_{\text {op }}$ or the third eigenfrequency from $6.23 \mathrm{~Hz}$ to $17.42 \mathrm{~Hz}$. Especially the blade flapping eigenfrequencies marked with $1,3,4,6$ and 9 rise strongly due to geometric stiffening. The dependency of the eigenfrequen-

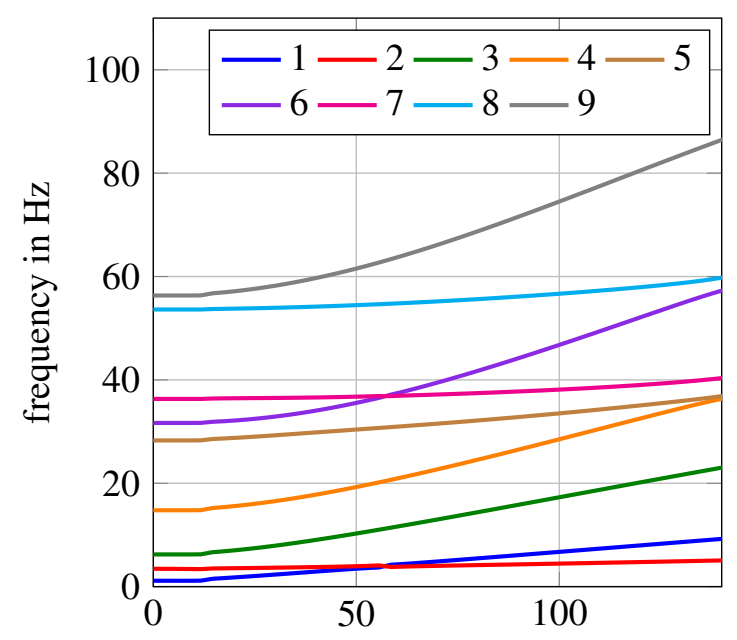

rotor speed in \% of nominal operational speed

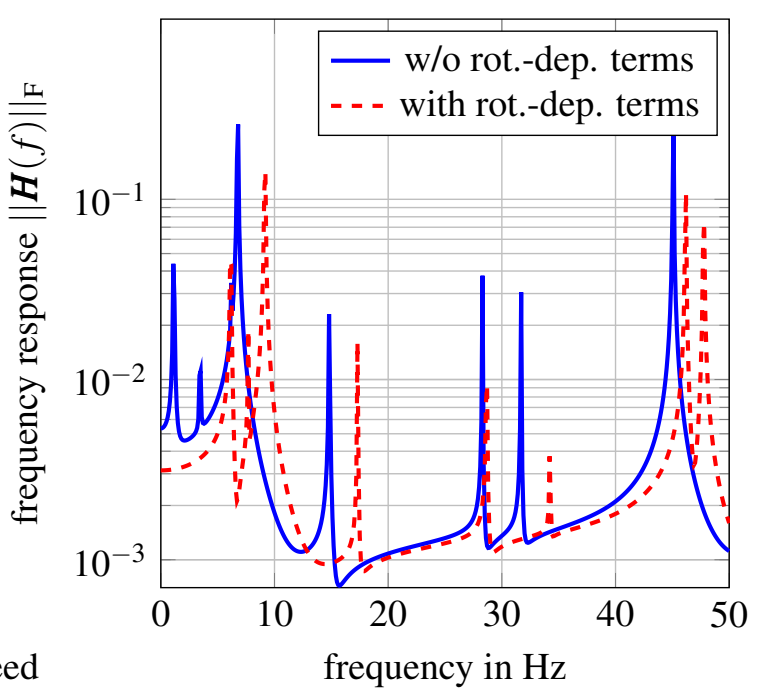

(b) Frobenius norm of the transfer function

Figure 3: Change of the dynamical behavior of a clamped rotor blade due to rotation (model provided by Airbus Helicopters) 
cies on the rotational velocity shows insistently that the dynamical behavior of the blade is strongly dependent on its rotation and, therefore, that the equations of motion are nonlinear in general.

In Figure $3 b$ the transfer function of two different models is compared. One is obtained from linear FEM, alone where the rotational dependence is ignored. The other model is also linear but contains the additional rotation-dependent terms at operational angular velocity. Input and output for the transfer function are both set at the tip of the blade, marked with (3) in Figure 2. It can be seen that both models behave very different. For a static load the rotating blade deforms less than the non-rotating blade which indicates the increase of stiffness due to geometric stiffening. With the rotation-dependent terms, the eigenfrequencies of the model change. Hereby, the amplitudes of the transfer function shift as well. Furthermore all amplitudes of the frequency response are lower for the stiffened rotating blade.

Both plots in Figure 3 demonstrate that the dynamical behavior of the blade is strongly dependent on its rotation and thus, that the rotation dependent terms have to be included in the equation of motion of the EMBS. For the reduction of the rotor and the study of the system input-output behavior it is thus not adequate to just use the non-rotating model obtained from linear FEM. Instead, the geometric stiffness matrix and other rotation-dependent terms from Equation (9) have to be included in the equations of motion of the rotating blade. In the following, 4 characteristic nodes are depicted for the study of the dynamical behavior as marked in Figure 2. They display the deformations at the fenestron hub (1), at the coupling point of rotor and airframe which is the main rotor hub (2), near the blade tip (3), and at the pilot's feet (4).

\section{RESULTS}

In this section different reduction approaches are compared for the helicopter model. Therefore, the relative transfer function error of the reduced system in Frobenius norm

$$
\varepsilon=\frac{\|\boldsymbol{H}(s)-\tilde{\boldsymbol{H}}(s)\|_{F}}{\|\boldsymbol{H}(s)\|_{F}}
$$

is calculated in the frequency band from 1 to $50 \mathrm{~Hz}$ with the Frobenius norm

$$
\|\boldsymbol{H}(s)\|_{F}=\sqrt{\operatorname{trace}\left(\boldsymbol{H}(s) \boldsymbol{H}^{H}(s)\right)} .
$$

\subsection{Reduction of Free Bodies}

At first the different reduction methods are compared for the free bodies rotor and airframe separately. An adequately reduced model of the separate bodies is a prerequisite for the combination in the coupled system. In Figure 4 the airframe and the rotor are reduced separately to orders around 100. The order of the reduced models is given in the legend in each case. Four different models are compared for the airframe and three models are compared for the rotor. The models are generated with the reduction techniques introduced in Subsection 2.2, model truncation (mod), moment matching with Krylov subspaces (Krylov) and POD. In addition to that, a reduction with an added surrogate mass is implemented for the airframe (mod am). This is done by generating the projection matrix with a model that contains an additional surrogate mass to represent the rotor that will be attached later. The model without surrogate mass is than reduced with the obtained projection matrix.

The modal truncation shows the poorest results with an error in the range of $10^{-3}$ to more than $10^{0}$. As described in [10], this is because a large number of eigenmodes is needed to account for local forces and, therefore, to describe the input-output behavior correctly. For the defined inputs and outputs the reduced airframe model generated with an added point mass reveals a smaller approximation error because the surrogate mass attached to the rotor shaft ensures to keep relevant shaft-bending modes in the reduced basis. 


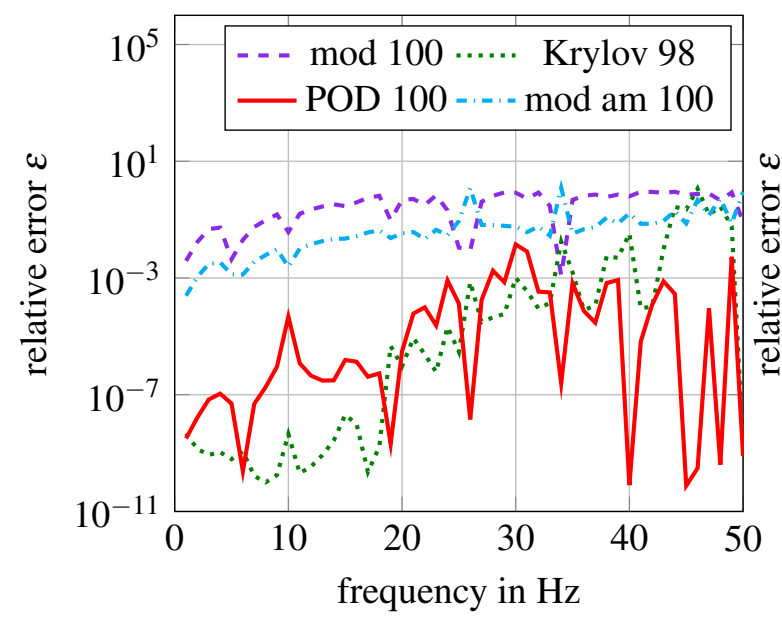

(a) airframe

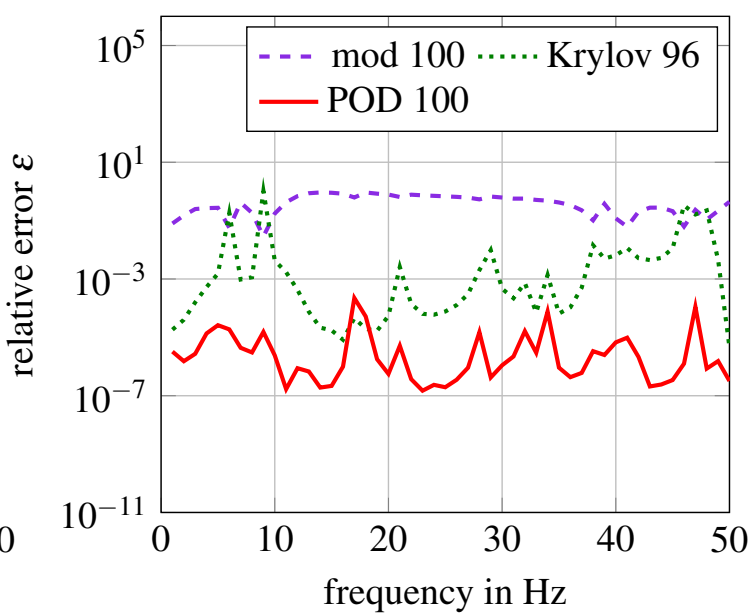

(b) rotor

Figure 4: Relative error for reduced bodies

The error for moment matching depends highly on the chosen shifts $\sigma_{\mathrm{k}}$. They are here set linearly spaced between 0 and $50 \mathrm{~Hz}$. For a reduction order of about 100, moment-matching displays a smaller error than the modal methods. The POD allows a small approximation error for both bodies and here never exceeds $10^{-2}$. The snapshots for POD are in this work linearly distributed in the range from 1 to $50 \mathrm{~Hz}$ with steps of $1 \mathrm{~Hz}$. According to the BSPR workflow, the reduced airframe and rotor can now be coupled to obtain the reduced system.

\subsection{Reduction of Coupled Elastic Multibody Systems}

Figure 5 shows the reduction errors for BSPR and SBR. With the exception of modal truncation, the comparison of both plots exhibits that the reduction errors for SBR are at least similarly small as those of BSPR. Modal truncation with added mass and CBM are techniques developed especially for SBR and, therefore, are not applicable in the BSPR framework and only included in the comparison of SBR. For both approaches the POD displays the smallest error.

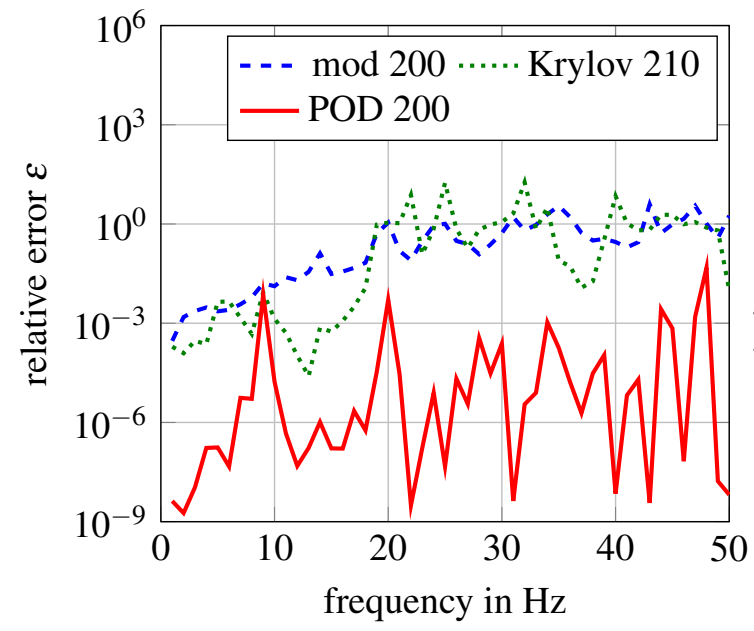

(a) BSPR

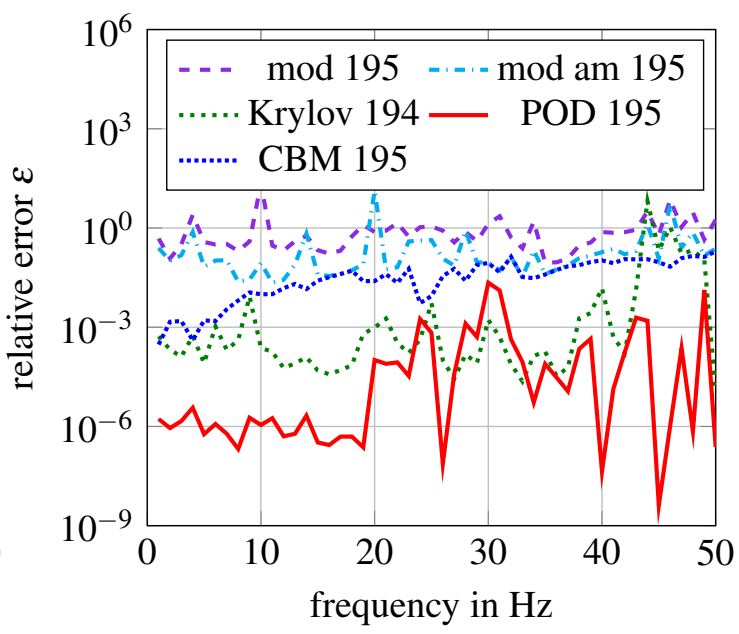

(b) SBR

Figure 5: Relative error for reduced system 
For the SBR, simple modal truncation reveals large errors. This is because the reduction of the bodies already induces a relatively large error as was shown in Subsection 5.1. In addition to that, modal truncation can neither account for the interaction between the different bodies and the dynamics induced by the coupling nor is it able to regard arbitrary dissipative forces, i.e., if $\boldsymbol{G}$ is not proportional to $\boldsymbol{M}$ and $\boldsymbol{K}$, which is the case if $\boldsymbol{G} \neq \mathbf{0}$, the eigenmodes may become complex. The approach outlined in Section 4, were a surrogate mass is attached to generate the reduced basis for the airfame is also presented in Figure 5b. The reduced model of the airframe with added mass is coupled to a reduced rotor obtained from modal truncation. The error for the resulting reduced coupled system is smaller than for the model generated with simple modal truncation but still relatively large. The modal truncation is also improved with the CBM by adding constraint modes which leads to a small error especially for low frequencies. Hence, both state of the art methods CBM and modal truncation with added mass can both improve the simple modal truncation. Nevertheless, the input-output based techniques, moment matching and POD, lead to even smaller approximation errors for the same reduction order. Especially the POD exhibits a good approximation to the full order transfer function. For the reduction order of 195, the maximal relative error for POD is $10^{-2}$ in contrast to $10^{-1}$ for CBM and $10^{1}$ for modal truncation with added mass.

It is another advantage of POD is that the reduction error scales down fast with increasing reduced orders. Figure 6 shows the relative errors for POD with SBR with reduced orders 95, 195 and 295. While the reduced model of order 95 exhibits a relatively large approximation error between $10^{-3}$ and $10^{\circ}$, the previously used model with 195 dof has a much smaller error especially for low frequencies. The approximation can be further improved by increasing the reduced order to 295 with the result that the error never exceeds $10^{-4}$. This offers the possibility to choose a model that is reduced to the respective requirements in terms of numerical efficiency and approximation quality. If one wishes for a smaller approximation error for the POD in Figure 5b, this can be reached by increasing the reduction order.

The reduction of the system from 285713 dof to only 195 dof enables much more efficient evaluations. The calculation of the transfer function at a single frequency point takes $56.4 \mathrm{CPU}$ seconds for the full system and only 0.05 seconds for the reduced one which is less than $0.1 \%$ and means an enormous speed-up.

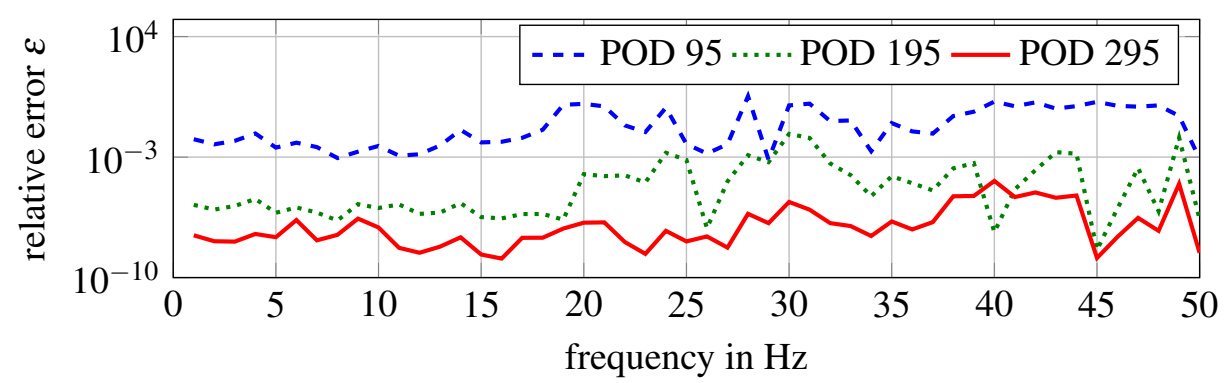

Figure 6: Relative error of POD with SBR for different reduction orders

\subsection{Comparison of Modeling Error and Reduction Error}

Two different possible errors were presented in Section 4 and Subsection 5.2. One arises from neglecting the rotation-dependent terms when formulating the equations of motion of a rotating body and is referred to here as modeling error. This error is prevented by adding these additional terms for a given constant angular velocity. The other error is the approximation error from reduction and is inevitable since a reduced model is necessary to enable efficient system evaluations. In this section both errors are contrasted. Therefore, the system is excited at the blade tip in vertical direction and the deformations at the output nodes (1), (2), (3) and (4) are computed. 


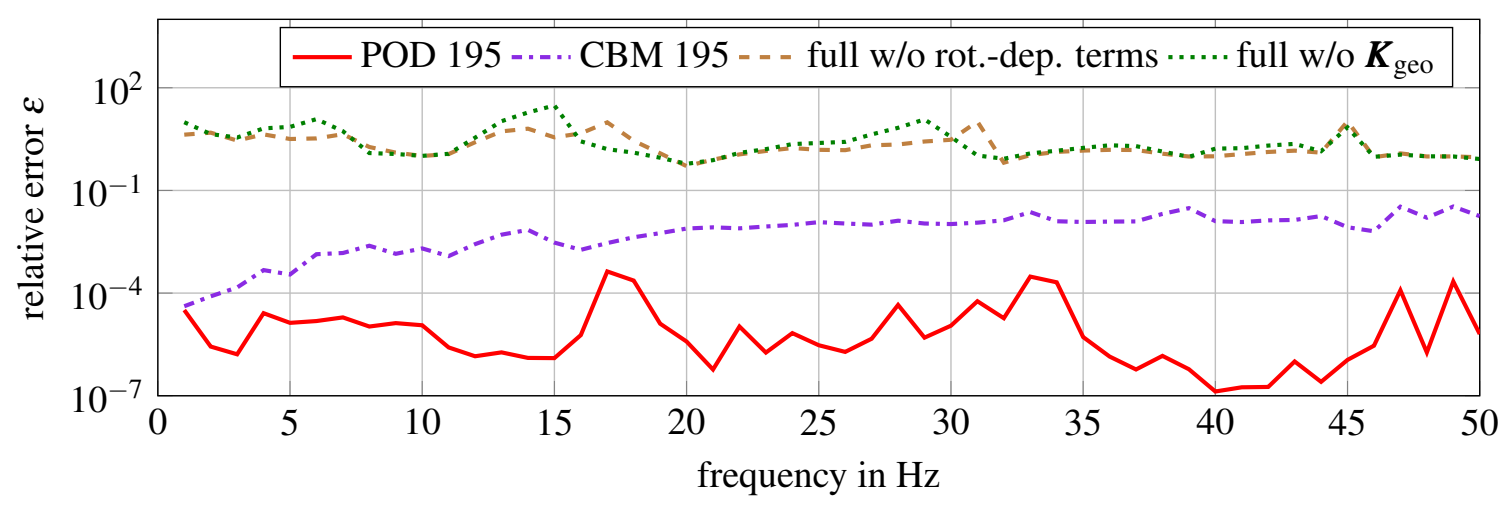

Figure 7: Influence of rotation dependent terms on vibration behavior

Figure 7 visualizes the relative error of the transfer functions for the different models related to the transfer function of the full-order model with rotation-dependent terms at nominal angular velocity. The errors of the reduced systems obtained from POD and CBM in the framework of SBR are confronted with the errors of two full-order models. One, called 'full w/o rot.-dep terms', neglects all rotation dependent terms and the other one, called 'full w/o $\boldsymbol{K}_{\text {geo }}$ ' is a model where geometric stiffness is neglected but all other rotation-dependent terms from Equation (9) are contained.

The error made when using the full non-rotating rotor model or a model without geometric stiffness is notable larger than the error of both reduced models on the presented frequency interval. Thus, all reduced models derived from the non-rotating model cannot serve as adequate reduced order model for the EMBS with fast rotating rotor. It can be seen, that especially the geometric stiffening of the body has large impact on the dynamical behavior. The reduced model generated with CBM with 195 dof approximates the full-order system better than both full-order models and exhibits low approximation errors i.e. for low frequencies. The POD again approximates the system with rotating rotor even better and has the smallest error over the whole frequency range. It reveals an maximal approximation error that is smaller in 4 orders of magnitude in comparison to the models without rotation-dependent terms.

\section{CONCLUSION}

This contribution presented a workflow for the reduction of Elastic Multibody Systems with rotating bodies. The obtained results improve the results from standard techniques highly. It was demonstrated that rotation dependent terms, i.e. geometric stiffness, have to be considered when modeling systems with fast rotating elastic bodies. Based on the linear description of a constantly rotating body with small deviations from reference configuration, projection based linear model order reduction could be applied. Especially the reduction of the bodies separately with proper orthogonal decomposition followed by a coupling of the reduced bodies allowed reduced models with low approximation errors. This was shown by consideration of the transfer function error of an industrial helicopter model. With the generated reduced, model the time needed to calculate the transfer function of the system, could be reduced by a factor greater than 1000 .

\section{Acknowledgments}

This research is done within the framework of the EVOLVE research project (FKZ 20A1902C) funded by the German Federal Ministry of Economics and Energy.
Gefördert durch:

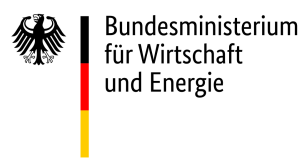

aufgrund eines Beschlusses des Deutschen Bundestages 


\section{REFERENCES}

[1] Craig, R.; Bampton, M.: Coupling of Substructures for Dynamic Analyses. AIAA Journal, Vol. 6, No. 7, pp. 1313-1319, 1968.

[2] Holzwarth, P.; Eberhard, P.: Input-Output Based Model Reduction for Interconnected Systems. In Oñate, E.; Oliver, J.; Huerta, A. (Eds.): Proceedings of the 5th European Conference of Computational Mechanics, Barcelona, 2014.

[3] Shabana, A.: Flexible Multibody Dynamics: Review of Past and Recent Developments. Multibody System Dynamics, Vol. 1, pp. 189-222, 1997.

[4] Schwertassek, R.; Wallrapp, O.: Dynamik flexibler Mehrkörpersysteme (in German). Braunschweig: Vieweg, 1999.

[5] Kirchgäßner, B.: Finite Elements in Rotordynamics. Procedia Engineering, Vol. 144, pp. 736-750, 2016.

[6] Nowakowski, C.: Zur Modellierung und Reduktion elastischer Bauteile unter verteilten Lasten für die Mehrkörpersimulation (in German). Dissertation, Schriften aus dem Institut für Technische und Numerische Mechanik der Universität Stuttgart, Vol. 35. Aachen: Shaker Verlag, 2014.

[7] Salimbahrami, S.B.: Structure Preserving Order Reduction of Large Scale Second Order Models. Dissertation, Technische Universität München. München, 2005.

[8] Fehr, J.: Automated and Error-Controlled Model Reduction in Elastic Multibody Systems. Dissertation, Schriften aus dem Institut für Technische und Numerische Mechanik der Universität Stuttgart, Vol. 21. Aachen: Shaker Verlag, 2011.

[9] Baur, U.; Beattie, C.; Benner, P.; Gugercin, S.: Interpolatory Projection Methods for Parameterized Model Reduction. SIAM Journal for Scientific Computing, Vol. 33, No. 5, pp. 2489-2518, 2011.

[10] Lehner, M.: Modellreduktion in elastischen Mehrkörpersystemen (in German). Dissertation, Schriften aus dem Institut für Technische und Numerische Mechanik der Universität Stuttgart, Vol. 10. Aachen: Shaker Verlag, 2007.

[11] Antoulas, A.: Approximation of Large-Scale Dynamical Systems. Philadelphia: SIAM, 2005.

[12] Kurz, T.; Eberhard, P.; Henninger, C.; Schiehlen, W.: From Neweul to Neweul-M²: Symbolical Equations of Motion for Multibody System Analysis and Synthesis. Multibody System Dynamics, Vol. 24, No. 1, pp. 25-41, 2010.

[13] MSCSoftware: MSC Nastran 2012 - Quick Reference Guide, 2011.

[14] Holzwarth, P.: Modellordnungsreduktion für substrukturierte mechanische Systeme (in German). Dissertation, Schriften aus dem Institut für Technische und Numerische Mechanik der Universität Stuttgart, Vol. 51. Aachen: Shaker Verlag, 2017.

[15] Bestle, P.; Eberhard, P.; Hanss, M.: Musical Instruments - Sound Synthesis of Virtual Idiophones. Journal of Sound Vibration, Vol. 395, pp. 187-200, 2017.

[16] Hasbun, M.; Saberi, H.; Blumenstein, R.: Modal Elastic Component Enhancements for RCAS. VFS International 76th Annual Forum \& Technology Display, 2020. 